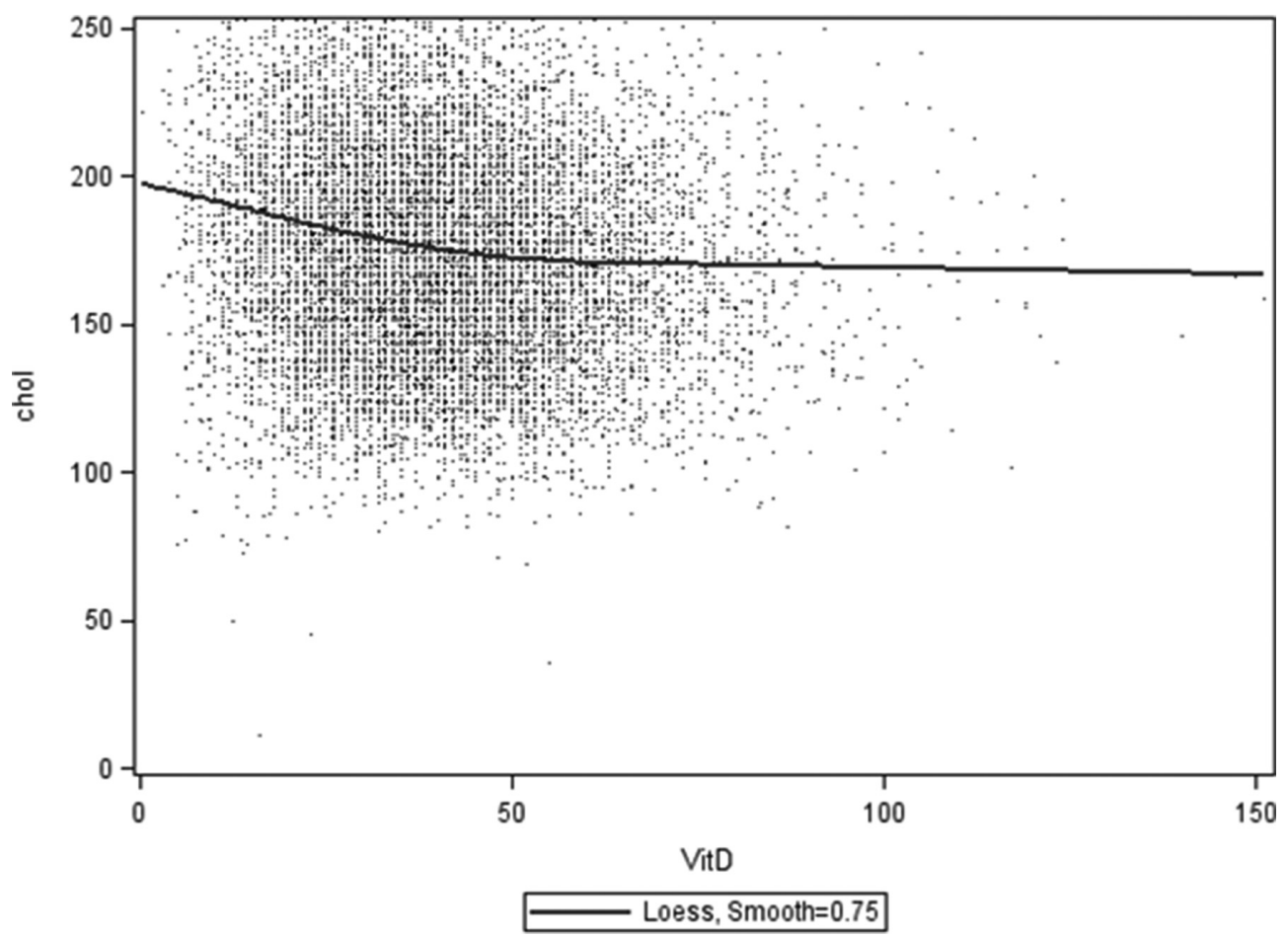

Abstract 455 Figure 1 Serum Cholestrol $(\mathrm{mg} / \mathrm{dl})$ vs Serum Vitamin D $(\mathrm{ng} / \mathrm{ml})$ with nonparametric estimate of the mean.

Abstract 455 Table 1 Slope of relationship between 25(OH)D and mean Cholesterol over difference ranges of $25(\mathrm{OH}) \mathrm{D}$.

\begin{tabular}{|c|c|c|c|c|}
\hline \multirow{2}{*}{$\begin{array}{l}\text { Range of } \\
25(\mathrm{OH}) \mathrm{D}\end{array}$} & \multicolumn{4}{|c|}{ Estimated slope ( $(55 \%$ Confidence Interval) } \\
\hline & Unadjusted & P.value & Adjusted & P.value \\
\hline $0.50 \mathrm{ng} / \mathrm{ml}$ & $-0.37(-0.42,-0.32)$ & $<0.0001$ & $.0 .30(-0.35,-0.24)$ & $<0.0001$ \\
\hline $50+n g l m l$ & $-0.09(-0.15,-0.02)$ & 0.014 & $\cdot 0.11(-0.18,-0.03)$ & 0.0037 \\
\hline
\end{tabular}

Conclusions Lupus Nephritis has higher IL-17 serum level and SLAM score compared to non lupus nephritis. There is significant correlation between IL-17 and SLAM score in LN but not significant in non $\mathrm{LN}$.

\section{ALGORITHMS TO IDENTIFY SYSTEMIC LUPUS ERYTHEMATOSUS (SLE) FROM ELECTRONIC HEALTH RECORD (EHR) DATA}

${ }^{1} \mathrm{R}$ Ramsey-Goldman*, ${ }^{2} \mathrm{~T}$ Walanus, ${ }^{3} \mathrm{~K}$ Jackson, ${ }^{1} \mathrm{~A}$ Chung, ${ }^{4} \mathrm{D}$ Erickson, ${ }^{1} \mathrm{~K}$ ManceraCuevas, ${ }^{5} \mathrm{~A}$ Kho. ${ }^{1}$ Division of Rheumatology, Medicine, Chicago, USA; ${ }^{2}$ Division of General Medicine and Geriatrics, Medicine, Chicago, USA; ${ }^{3}$ Center for Health Information, Medicine, Chicago, USA; ${ }^{4}$ Preventive Medicine, Preventive Medicine, Chicago, USA; ${ }^{5}$ General Medicine, Medicine, Chicago, USA
Background and aims Background: SLE is difficult to diagnose because of the diverse manifestations occurring over time and across care sites. Electronic health records (EHR) present a rich source of patient information which can be mined for diagnosis and identification to improve quality of care or to enrol patients in studies.

Aim Build a rule-based algorithm for each revised 1982/1997 ACR classification criteria for SLE using EHR data.

Methods We included patients from the Chicago Lupus Database (CLD) fulfilling 4 or more of the ACR classification criteria for SLE who also had records in the Northwestern Medicine Electronic Data Warehouse (NMEDW) EHR. ICD-9 codes and lab test results for each ACR SLE criterion were ascertained. We queried patient diagnoses, lab results and used a simple chart string for lab test results from physician notes. Results Data from 515/783 patients in CLD and the NMEDW EHR were included. When using ICD 9 codes only $8.8 \%$ of patients from CLD/NMEDW were identified. With the addition of lab results to the query concordance increased to $54.7 \%$, and a simple text string query to search physician notes for additional lab results increased identification to $57.5 \%$.

Conclusion Using ICD codes plus laboratory data from NMEDW increased fulfilment of classification criteria but is still not optimal for patient identification. Additional strategies such as using natural language processing (NLP) or examining fulfilment of SLICC classification criteria for SLE which includes more lab results than ACR may yield an improved rule-based algorithm for the identification of SLE patients in EHR data. 